ESAIM: M2AN

Vol. 40, No 5, 2006, pp. 923-937

DOI: $10.1051 / \mathrm{m} 2 \mathrm{an}: 2006040$
ESAIM: Mathematical Modelling and Numerical Analysis

www.edpsciences.org/m2an

\title{
MAXIMUM-NORM RESOLVENT ESTIMATES FOR ELLIPTIC FINITE ELEMENT OPERATORS ON NONQUASIUNIFORM TRIANGULATIONS
}

\author{
Nikolai Yu. Bakaev ${ }^{1}$, Michel Crouzeix ${ }^{2}$ and Vidar Thomée ${ }^{3}$
}

\begin{abstract}
In recent years several papers have been devoted to stability and smoothing properties in maximum-norm of finite element discretizations of parabolic problems. Using the theory of analytic semigroups it has been possible to rephrase such properties as bounds for the resolvent of the associated discrete elliptic operator. In all these cases the triangulations of the spatial domain has been assumed to be quasiuniform. In the present paper we show a resolvent estimate, in one and two space dimensions, under weaker conditions on the triangulations than quasiuniformity. In the two-dimensional case, the bound for the resolvent contains a logarithmic factor.
\end{abstract}

Mathematics Subject Classification. 65M06, 65M12, 65M60.

Received: May 2, 2006.

\section{INTRODUCTION}

Consider the initial-value problem

$$
u_{t}-\Delta u=0 \quad \text { in } \Omega \quad \text { and } u=0 \quad \text { on } \partial \Omega, \quad \text { for } t>0, \quad \text { with } u(\cdot, 0)=v \quad \text { in } \Omega,
$$

where $\Omega$ is a domain in $\mathbb{R}^{2}$, and denote by $E(t)$ the solution operator related to this problem and defined by $u(t)=E(t) v$. Then it is a special case of a result of Stewart [11] that if $\partial \Omega$ is smooth, then $E(t)$ is an analytic semigroup on $\mathcal{C}_{0}(\bar{\Omega})=\{v \in \mathcal{C}(\bar{\Omega}): v=0$ on $\partial \Omega\}$ generated by $\Delta$. This follows from the resolvent estimate

$$
\left\|(\lambda I+\Delta)^{-1} v\right\|_{\mathcal{C}} \leq \frac{C}{1+|\lambda|}\|v\|_{\mathcal{C}}, \quad \text { for } v \in \mathcal{C}_{0}(\bar{\Omega}) \quad \text { and } \lambda \notin \Sigma_{\delta}=\{\lambda:|\arg \lambda| \leq \delta\}
$$

\footnotetext{
Keywords and phrases. Resolvent estimates, stability, smoothing, maximum-norm, elliptic, parabolic, finite elements, nonquasiuniform triangulations.

1 Department of Economic Dynamics, EAI, Moscow Engineering Physics Institute (State University), Kashirskoe Shosse 31, Moscow 115409, Russia. bakaev@postman.ru

2 IRMAR, Université de Rennes I, Campus de Beaulieu, 35042 Rennes Cedex, France. michel.crouzeix@univ-rennes1.fr

3 Department of Mathematics, Chalmers University of Technology, 41296 Göteborg, Sweden. thomee@math.chalmers.se
} 
where $\|v\|_{\mathcal{C}}=\sup _{x \in \Omega}|v(x)|$ and where $\delta \in\left(0, \frac{1}{2} \pi\right)$ is arbitrary. In addition to the stability estimate $\|E(t) v\|_{\mathcal{C}} \leq$ $\|v\|_{\mathcal{C}}$, which follows by the maximum-principle, this entails the smoothing estimate

$$
\left\|E^{\prime}(t) v\right\|_{\mathcal{C}} \leq \frac{C}{t}\|v\|_{\mathcal{C}}, \quad \text { for } v \in \mathcal{C}_{0}(\bar{\Omega})
$$

Such a result is valid also under lesser regularity requirements on $\partial \Omega$, $c f$. Ouhabaz [8].

In this paper, we are interested in maximum-norm estimates for spatially semidiscrete approximations of parabolic problems such as (0.1) based on continuous, piecewise polynomial finite elements of degree $r-1 \geq 1$. Let $\mathcal{T}_{h}=\{\tau\}$ denote a family of closed face-to-face triangles in $\bar{\Omega}$ with mutually disjoint interiors, with diameter $h_{\tau}$, and set $h=\max _{\tau \in \mathcal{T}_{h}} \operatorname{diam}(\tau)$. Let $\Omega_{h}$ be the interior of the set $\cup\left\{\tau: \tau \in \mathcal{T}_{h}\right\}$ and assume that $\Omega_{h} \subseteq \Omega$. If $\Omega$ is a polygonal domain it is natural to choose $\mathcal{T}_{h}$ so that $\Omega_{h}=\Omega$.

We consider, in fact, a whole family of such triangulations $\left\{\mathcal{T}_{h}\right\}$ and assume that this is a regular family of triangulations in the sense that $h_{\tau} / d_{\tau} \leq C$ for all $\tau \in \mathcal{T}_{h}$, where $d_{\tau}$ is the radius of the largest disc contained in $\tau$. We associate with $\mathcal{T}_{h}$ the finite dimensional spaces

$$
S_{h}=\left\{\chi \in \mathcal{C}(\bar{\Omega}):\left.\chi\right|_{\tau} \in P_{r-1} \text { for } \tau \in \mathcal{T}_{h}, \chi=0 \text { on } \partial \Omega \cup\left(\Omega \backslash \Omega_{h}\right)\right\},
$$

where $P_{k}$ denotes the set of polynomials of degree $k$.

The semidiscrete finite element problem associated with (0.1) is then to find $u_{h}(t) \in S_{h}$ for $t>0$ such that, with $v_{h} \in S_{h}$ given,

$$
\begin{aligned}
\left(u_{h, t}, \chi\right)+\left(\nabla u_{h}, \nabla \chi\right)=0 & \text { for } \chi \in S_{h}, t>0, \\
u_{h}(\cdot, 0)=v_{h} & \text { in } \Omega, \quad \text { where }(v, w)=\int_{\Omega} v(x) \overline{w(x)} \mathrm{d} x .
\end{aligned}
$$

With $-\Delta_{h}: S_{h} \rightarrow S_{h}$ defined by

$$
-\left(\Delta_{h} \psi, \chi\right)=(\nabla \psi, \nabla \chi), \quad \forall \psi, \chi \in S_{h},
$$

this problem may also be written

$$
u_{h, t}-\Delta_{h} u_{h}=0, \quad \text { for } t>0, \quad \text { with } u_{h}(0)=v_{h} .
$$

The solution operator of this problem, defined by $u_{h}(t)=E_{h}(t) v_{h}$, is the semigroup $E_{h}(t)=\mathrm{e}^{\Delta_{h} t}$ in $S_{h}$ generated by $\Delta_{h}$. The issue is then to show that this semigroup is analytic in $S_{h}$, equipped with the maximumnorm, and this may be expressed either as a resolvent estimate for $-\Delta_{h}$ or as the stability and a smoothing property of $E_{h}(t)$.

In Schatz et al. [9] it was thus shown in the case of a convex domain $\Omega$ with smooth boundary, and for quasiuniform piecewise linear finite elements $(r=2)$ that, with $\ell_{h}=\max (1, \log (1 / h))$,

$$
\left\|E_{h}(t) v_{h}\right\|_{\mathcal{C}}+t\left\|E_{h}^{\prime}(t) v_{h}\right\|_{\mathcal{C}} \leq C \ell_{h}\left\|v_{h}\right\|_{\mathcal{C}}, \quad \text { for } v_{h} \in S_{h} .
$$

Using semigroup theory this shows the resolvent estimate (cf. [12], Lem. 8.7)

$$
\left\|\left(\lambda I+\Delta_{h}\right)^{-1} v_{h}\right\|_{\mathcal{C}} \leq \frac{C \ell_{h}^{2}}{1+|\lambda|}\left\|v_{h}\right\|_{\mathcal{C}}, \quad \text { for } \lambda \notin \Sigma_{\delta_{h}}, \quad \text { where } \delta_{h}=\frac{1}{2} \pi-c \ell_{h}^{-2} .
$$

In Schatz et al. [10] the logarithmic factor in (0.4) was removed, which implies that the resolvent estimate (0.5) holds without a logarithmic factor as well, and for $\lambda \notin \Sigma_{\delta}$, for some $\delta \in\left(0, \frac{1}{2} \pi\right)$ independent of $h$. In Bakaev et al. [3] a direct proof was given that this resolvent estimate holds for any angle $\delta \in\left(0, \frac{1}{2} \pi\right)$. The result in [3] 
holds for $\Omega$ in $R^{d}$ with $d \geq 2$ arbitrary, with $\partial \Omega$ smooth. In Chatzipantelidis et al. [4] such a resolvent estimate, with a logarithmic factor, was shown when $\Omega$ is a plane polygonal domain, which may be nonconvex. For some earlier work $c f .$, e.g., $[1,2,7]$.

In all these results quoted the family of triangulations is required to be quasiuniform, which is a somewhat undesirable restriction. Our purpose in this paper is therefore to weaken this condition. The technique of proof will depend heavily on Crouzeix and Thomée [5], where the stability of the $L_{2}$-projection onto $S_{h}$ was studied under milder assumptions on the triangulations than quasiuniformity.

An earlier attempt to treat this problem was made in Crouzeix and Thomée [6] where a resolvent estimate of the desired type, with a logarithmic factor, was shown for a modified discrete Laplacian, defined by

$$
-\left(\Delta_{h} \psi, \chi\right)_{h}=(\nabla \psi, \nabla \chi), \quad \forall \psi, \chi \in S_{h}
$$

where $(\cdot, \cdot)_{h}$ denotes a simple quadrature approximation of the $L_{2}$-inner product, and for triangulations of Delaunay type, not required to be quasiuniform.

We now introduce some notation. Following [5], given $\tau_{0} \in \mathcal{T}_{h}$, we let $Q_{j}\left(\tau_{0}\right)$ denote the set of triangles which are " $j$ triangles away from $\tau_{0}$ ", defined by setting $Q_{0}\left(\tau_{0}\right)=\tau_{0}$ and then, recursively, for $j \geq 1, Q_{j}\left(\tau_{0}\right)$ to be the union of the closed triangles $\tau$ which are not in $\bigcup_{i<j} Q_{i}\left(\tau_{0}\right)$, but which have at least one vertex in $Q_{j-1}\left(\tau_{0}\right)$. We further set $l\left(\tau_{0}, \tau\right)=j$ for $\tau \in Q_{j}\left(\tau_{0}\right)$ and denote by $n_{j}\left(\tau_{0}\right)$ the number of triangles in $Q_{j}\left(\tau_{0}\right)$.

In what follows we shall use the following auxiliary result from [5] showing the exponential decay property of the $L_{2}$-projection $P_{h}$ which was used to show the maximum-norm stability of this operator:

Lemma 0.1. There exist $C>0$ and $\gamma=\gamma_{r} \in(0,1)$ such that, for all $\tau, \tau_{0} \in \mathcal{T}_{h}$ and $v \in L_{2}$, with supp $v \in \tau_{0}$,

$$
\left\|P_{h} v\right\|_{L_{2}(\tau)} \leq C \gamma^{l\left(\tau, \tau_{0}\right)}\|v\|_{L_{2}}
$$

In [5] it was shown that one can choose, e.g., $\gamma_{2}=0.318, \gamma_{3}=0.376, \gamma_{4}=0.353$.

We now make the assumption that the family $\left\{\mathcal{T}_{h}\right\}$ of triangulations satisfies, with some $\alpha \geq 1$ and $\beta \geq 1$,

$$
h_{\tau} / h_{\tau_{0}} \leq C \alpha^{l\left(\tau, \tau_{0}\right)}, \quad \text { for all } \tau, \tau_{0} \in \mathcal{T}_{h}
$$

and

$$
n_{j}(\tau) \leq C \beta^{j}, \quad j \geq 1, \quad \text { for all } \tau \in \mathcal{T}_{h}
$$

For quasiuniform triangulations this holds with $\alpha=1$ and $\beta$ any number $>1$, and if $(0.6)$ holds with $\alpha>1$, we may choose $\beta=\alpha^{4}$ in (0.7).

Under these assumptions we show that if the above conditions on $\left\{\mathcal{T}_{h}\right\}$ hold, with $(0.6)$ and $(0.7)$, and if

$$
\alpha^{2} \beta \gamma<1
$$

with $\gamma$ as in Lemma 0.1 , then, for any fixed $\delta \in\left(0, \frac{1}{2} \pi\right)$, we have

$$
\left\|\left(\lambda I+\Delta_{h}\right)^{-1} \chi\right\|_{\mathcal{C}} \leq \frac{C \ell_{h}^{1 / 2}}{1+|\lambda|}, \quad \forall \chi \in S_{h}, \quad \lambda \notin \Sigma_{\delta} .
$$

Here and below we write $\ell_{h}=\max \left(1, \log \left(1 / h_{\text {min }}\right)\right)$, where $h_{\text {min }}=\min _{\tau \in \mathcal{T}_{h}} h_{\tau}$. For example, for $r=2$, with $\beta=\alpha^{4}$, the condition $(0.8)$ requires $\alpha<\gamma_{2}^{-1 / 6}=(0.318)^{-1 / 6} \approx 1.21$, which permits a substantial degree of nonquasiuniformity.

We note that the $L_{2}$-projection $P_{h}: L_{2} \rightarrow S_{h}$ is stable in maximum-norm if $\alpha \beta \gamma<1$, thus in particular when condition (0.8) holds. This was shown in [5] in the case of a polygonal domain $\Omega$, with $\Omega_{h}=\bar{\Omega}$, but the proof is valid under our present assumptions.

It follows from (0.9) by standard semigroup theory that, under our present assumptions on $\mathcal{T}_{h}$, the solution operator $E_{h}(t)$ of (0.3) satisfies the stability and smoothing estimate (0.4), with the factor $\ell_{h}$ replaced by $\ell_{h}^{1 / 2}$. 
The resolvent estimate (0.9) will be shown in Section 3 below, in which the Laplacian is replaced by a more general second order elliptic operator. We begin in the next Section 2 by considering a spatially one-dimensional elliptic operator. In this case we shall show the corresponding resolvent estimate without the logarithmic factor.

\section{The One-Dimensional Case}

In this section we consider the one-dimensional elliptic operator

$$
A u=-\left(a u^{\prime}\right)^{\prime}+b u^{\prime}+c u, \quad \text { in } \Omega=(0,1)
$$

with $a, b, c$ bounded real-valued functions, with $a(x) \geq a_{0}>0$ on $\Omega$. We introduce the sesquilinear form

$$
A(u, w)=\int_{0}^{1}\left(a u^{\prime} \bar{w}^{\prime}+b u^{\prime} \bar{w}+c u \bar{w}\right) \mathrm{d} x .
$$

It is then an easy matter to show that there exist constants $c_{0}>0, c_{1}, c_{2}, c_{3} \in \mathbb{R}$ such that

$$
c_{0}\left\|w^{\prime}\right\|^{2}-c_{1}\|w\|^{2} \leq \operatorname{Re} A(w, w) \leq c_{2}\left\|w^{\prime}\right\|^{2} \quad \text { and } \quad|\operatorname{Im} A(w, w)| \leq c_{3}\left\|w^{\prime}\right\|\|w\|, \quad \forall w \in H_{0}^{1} .
$$

Here $\|\cdot\|$ denotes the usual $L_{2}$-norm on $\Omega$. With the sesquilinear form (1.1) we associate its numerical range $W(A) \subset \mathbb{C}$ defined by

$$
W(A)=\left\{A(w, w) ; w \in H_{0}^{1},\|w\|=1\right\} .
$$

From the previous assumptions we may write $A(w, w)=x+\mathrm{i} y$ for $\|w\|=1$, where $x \geq c_{0}\left\|w^{\prime}\right\|^{2}-c_{1}$ and $|y| \leq c_{3}\left\|w^{\prime}\right\|$. Therefore

$$
W(A) \subset \mathcal{P}=\left\{z=x+\mathrm{i} y \in \mathbb{C} ; x \geq c_{0} c_{3}^{-2} y^{2}-c_{1}\right\},
$$

e.g., the numerical range of $A$ is included in the horizontal parabolic domain $\mathcal{P}$.

We consider now a closed subset $\Sigma \subset \mathbb{C}$ of the complex plane such that

$$
d(\lambda, \mathcal{P}) \geq c(1+|\lambda|), \quad \text { for all } \lambda \in \Sigma, \quad \text { where } c>0
$$

For instance, we can choose for $\Sigma$ the complement of any open sector containing $\mathcal{P}$. When $A$ is selfadjoint positive definite, $\mathcal{P}$ is a subset of the positive real axis, and $\Sigma$ may be chosen as the complement of any sector $\Sigma_{\delta}$ as defined in $(0.2)$.

Let $0=x_{0}<x_{1}<\cdots<x_{N+1}=1$ be a partition of $\Omega$ into subintervals $I_{j}=\left(x_{j}, x_{j+1}\right)$ and let $h_{j}=x_{j+1}-x_{j}$. We assume

$$
h_{i} / h_{j} \leq C \alpha^{|i-j|}, \quad \text { with } \alpha \geq 1
$$

Let $S_{h}=\left\{\chi \in \mathcal{C}_{0}(\Omega):\left.\chi\right|_{I_{j}} \in P_{r-1}, j=0, \ldots, N\right\}$, where $P_{k}$ denotes the set of polynomials of degree $\leq k$, and define $A_{h}: S_{h} \rightarrow S_{h}$ by

$$
\left(A_{h} \psi, \chi\right)=A(\psi, \chi), \quad \forall \psi, \chi \in S_{h} .
$$

The following is then the main result in this section.

Theorem 1. Under the above assumptions, with $1 \leq \alpha<r$, we have

$$
\left\|\left(\lambda I-A_{h}\right)^{-1} v_{h}\right\|_{\mathcal{C}} \leq \frac{C}{1+|\lambda|}\left\|v_{h}\right\|_{\mathcal{C}}, \quad \forall \lambda \in \Sigma, v_{h} \in S_{h} .
$$

Proof. We introduce, for $x \in \Omega$, the adjoint discrete Green's function

$$
G_{h}^{x}(y, \bar{\lambda})=\left(\left(\bar{\lambda} I-A_{h}^{*}\right)^{-1} \delta_{h}^{x}\right)(y), \quad \text { for } \lambda \in \Sigma,
$$


where $\delta_{h}^{x} \in S_{h}$ is the discrete delta-function defined by

$$
\left(\chi, \delta_{h}^{x}\right)=\chi(x), \quad \forall \chi \in S_{h}
$$

It is easy to see that

$$
\left(\left(\lambda I-A_{h}\right)^{-1} \chi\right)(x)=\left(\chi, G_{h}^{x}(., \bar{\lambda})\right), \quad \forall \chi \in S_{h},
$$

and in order to prove Theorem 1 it suffices to show that, with $C$ independent of $x$ and $\lambda$,

$$
\left\|G_{h}^{x}(., \bar{\lambda})\right\|_{L_{1}} \leq \frac{C}{1+|\lambda|}, \quad \text { for } \lambda \in \Sigma
$$

The following will be a basic tool.

Lemma 1.1. There is a constant $C=C_{\Sigma}$ such that, for $v \in H_{0}^{1}$ and $\lambda \in \Sigma$,

$$
\text { if } \quad \lambda\|v\|^{2}-A(v, v)=F, \quad \text { then } \quad(1+|\lambda|)\|v\|^{2}+\left\|v^{\prime}\right\|^{2} \leq C|F| .
$$

Proof. We first note that, since $A(v, v) /\|v\|^{2} \in W(A)$, we have

$$
d(\lambda, \mathcal{P})\|v\|^{2} \leq\left|\lambda-A(v, v) /\|v\|^{2}\right|\|v\|^{2}=|F| .
$$

By (1.5) this shows

$$
(1+|\lambda|)\|v\|^{2} \leq C|F|
$$

The conclusion of the lemma follows since, by (1.2) and the triangle inequality,

$$
c_{0}\left\|v^{\prime}\right\|^{2} \leq \operatorname{Re} A(v, v)+c_{1}\|v\|^{2} \leq|F|+\left(c_{1}+\operatorname{Re} \lambda\right)\|v\|^{2} \leq C|F| .
$$

We note that, with $G=G_{h}^{x}(\cdot, \bar{\lambda})$ for $x \in \Omega, \lambda \in \Sigma$, we have

$$
\lambda(\chi, G)-A(\chi, G)=\left(\chi, \delta_{h}^{x}\right)=\chi(x), \quad \forall \chi \in S_{h} .
$$

Choosing $\chi=G$ and using Lemma 1.1 we obtain

$$
(1+|\lambda|)\|G\|^{2}+\left\|G^{\prime}\right\|^{2} \leq C\|G\|_{\mathcal{C}} \leq C\|G\|^{1 / 2}\left\|G^{\prime}\right\|^{1 / 2} .
$$

Using the inequality $x y \leq \frac{1}{4} x^{4}+\frac{3}{4} y^{4 / 3}$ to bound the right hand side, we find

$$
(1+|\lambda|)\|G\|^{2}+\left\|G^{\prime}\right\|^{2} \leq \frac{1}{2}\left\|G^{\prime}\right\|^{2}+C\|G\|^{2 / 3},
$$

and hence

$$
\|G\| \leq \frac{C}{(1+|\lambda|)^{3 / 4}} \quad \text { and } \quad\left\|G^{\prime}\right\| \leq \frac{C}{(1+|\lambda|)^{1 / 4}} .
$$

Since $\|G\|_{L_{1}} \leq\|G\|$ this implies (1.7) for $\lambda$ bounded.

For treating large values of $\lambda \in \Sigma$ we use the weight function

$$
\rho(y)=\rho_{h}^{x}(y)=\left((x-y)^{2}+h_{x}^{2}\right)^{1 / 2}, \quad \text { where } h_{x}:=h_{j} \quad \text { if } x \in\left[x_{j}, x_{j+1}\right) .
$$

We consider the expression

$$
\lambda\|\rho G\|^{2}-A(\rho G, \rho G)=\lambda\left(\rho^{2} G, G\right)-A\left(\rho^{2} G, G\right)-R(G, G)
$$


where

$$
R(G, G)=A(\rho G, \rho G)-A\left(\rho^{2} G, G\right),
$$

or, after subtraction of (1.8) with $\chi=P_{h}\left(\rho^{2} G\right)$,

$$
\lambda\|\rho G\|^{2}-A(\rho G, \rho G)=F,
$$

where

$$
F=-A\left(\rho^{2} G-P_{h}\left(\rho^{2} G\right), G\right)-\left(\rho^{2} G, \delta\right)-R(G, G) .
$$

By Lemma 1.1 this implies

$$
(1+|\lambda|)\|\rho G\|^{2}+\left\|(\rho G)^{\prime}\right\|^{2} \leq C|F| .
$$

The proof of the bound needed for the right hand side will be based on several lemmas. The first one is a one-dimensional analogue of Lemma 0.1 .

Lemma 1.2. There exists $C>0$ such that, for all $v \in L_{2}$ with $\operatorname{supp}(v) \in I_{l}$,

$$
\left\|P_{h} v\right\|_{L_{2}\left(I_{j}\right)} \leq C \gamma^{|j-l|}\|v\|, \quad \text { for all } j, l, \quad \text { where } \gamma=\gamma_{r}=1 / r .
$$

Proof. We recall some material from [5]. First we introduce the spaces $S_{h}^{2}=\left\{\chi \in S_{h} ; \chi\left(x_{j}\right)=0, j=1, \ldots, N\right\}$ and $S_{h}^{1}$, the orthogonal complement of $S_{h}^{2}$ in $S_{h}$ with respect to the inner product in $L_{2}(\Omega)$. For $r=2$ we have $S_{h}^{2}=\{0\}$ and $S_{h}^{1}=S_{h}$. We also introduce the orthogonal projection $\pi_{j}$ onto $S_{h}^{j}, j=1,2$, and obtain at once

$$
P_{h}=\pi_{1}+\pi_{2} .
$$

Recall that $\pi_{2}$ is determined locally on each $I_{j}$ by the equations

$$
\left(\pi_{2} w, q\right)_{L_{2}\left(I_{j}\right)}=(w, q)_{L_{2}\left(I_{j}\right)}, \quad \text { for all } q \in P_{r-1} \quad \text { with } q\left(x_{j}\right)=q\left(x_{j+1}\right)=0 .
$$

Thus, since $\operatorname{supp}(v) \subset I_{l}$, we have, since then $\left.\pi_{2} v\right|_{I_{j}}=0$, that

$$
\left\|P_{h} v\right\|_{L_{2}\left(I_{j}\right)}=\left\|\pi_{1} v\right\|_{L_{2}\left(I_{j}\right)} \text { if } j \neq l,
$$

and also

$$
\left\|P_{h} v\right\|_{L_{2}\left(I_{l}\right)} \leq\left\|P_{h} v\right\| \leq\|v\| .
$$

To show (1.14) it therefore suffices to consider the case $j \neq l$.

We now consider the functions $\psi_{i}, i=1, \ldots, N$, defined by $\psi_{i} \in S_{h}^{1}$ and $\psi_{i}\left(x_{j}\right)=\delta_{i j}$ for $j=1, \ldots, N$. Recall from Lemma 2 of [5] that $\operatorname{supp}\left(\psi_{j}\right)=I_{j-1} \cup I_{j}$, and that these functions constitute a basis for $S_{h}^{1}$ with

$$
\left\|\psi_{i+1}\right\|_{L_{2}\left(I_{i}\right)}^{2}=\left\|\psi_{i}\right\|_{L_{2}\left(I_{i}\right)}^{2}=\frac{h_{i}}{r^{2}-1}, \quad\left\|\psi_{i}\right\|^{2}=\frac{h_{i-1}+h_{i}}{r^{2}-1} \quad \text { and } \quad\left(\psi_{i}, \psi_{i+1}\right)=(-1)^{r} \frac{h_{i}}{r\left(r^{2}-1\right)} .
$$

Now if we set $\pi_{1} v=\sum_{i=1}^{N} w_{i} \psi_{i}$, we have, with $w_{0}=w_{N+1}=0$,

$$
\left(\psi_{i-1}, \psi_{i}\right) w_{i-1}+\left\|\psi_{i}\right\|^{2} w_{i}+\left(\psi_{i+1}, \psi_{i}\right) w_{i+1}=\left(v, \psi_{i}\right), \quad \text { for } i=1, \ldots, N .
$$

After division of the $i$ th equation by $\left\|\psi_{i}\right\|^{2}$, this linear system can be written as

$$
(I+K) W=F:=\left(f_{1}, \ldots, f_{N}\right)^{T}, \quad \text { with } f_{i}=\left(v, \psi_{i}\right) /\left\|\psi_{i}\right\|^{2},
$$


where $W=\left(w_{1}, \ldots, w_{N}\right)^{T}$ and where we note that $f_{i}=0$ for $i \neq l, l+1$. Here $K=\left(k_{i j}\right)$ is the tridiagonal $N \times N$ matrix with diagonal entries $k_{i i}=0$ and bidiagonal elements

$$
k_{i, i-1}=\frac{\left(\psi_{i}, \psi_{i-1}\right)}{\left\|\psi_{i}\right\|^{2}}=\frac{(-1)^{r}}{r} \frac{h_{i-1}}{h_{i-1}+h_{i}} \quad \text { and } \quad k_{i, i+1}=\frac{\left(\psi_{i}, \psi_{i+1}\right)}{\left\|\psi_{i}\right\|^{2}}=\frac{(-1)^{r}}{r} \frac{h_{i}}{h_{i-1}+h_{i}} .
$$

We now introduce the norms

$$
\|W\|_{p}=\left(\sum_{i=1}^{N}\left(h_{i+1}+h_{i}\right)\left|w_{i}\right|^{p}\right)^{1 / p}, \quad \text { for } 1 \leq p<\infty, \quad \text { with }\|W\|_{\infty}=\max _{i}\left|w_{i}\right|,
$$

and also denote by $\|\cdot\|_{p}$ the matrix operator norms induced by these vector norms. In particular we have $\|K\|_{\infty}=\max _{i} \sum_{j}\left|k_{i j}\right|=1 / r$, and noticing that $D K D^{-1}=K^{T}$, where $D=\operatorname{diag}\left(h_{0}+h_{1}, h_{1}+h_{2}, \ldots, h_{N-1}+h_{N}\right)$, we then also obtain $\|K\|_{1}=1 / r$. From the Riesz-Thorin interpolation theorem we deduce that $\|K\|_{p} \leq 1 / r$ for all $p$ with $1 \leq p \leq \infty$.

We now introduce the projection $P_{j}: \mathbb{C}^{N} \rightarrow \mathbb{C}^{N}$ defined by $\left(P_{j} W\right)_{i}=w_{i}$ if $i=j-1$ or $i=j$, and $=0$ otherwise. Using (1.15) and the $(2 s+1)$-diagonal character of $K^{s}$ we find

$$
P_{j} W=\sum_{s \geq|j-l|-1}(-1)^{s} P_{j} K^{s} F,
$$

and therefore

$$
\left\|P_{j} W\right\|_{2} \leq \sum_{s \geq|j-l|-1}\|K\|_{2}^{s}\|F\|_{2} \leq \frac{1}{r^{|j-l|}} \frac{r^{2}}{r-1}\|F\|_{2} .
$$

Simple calculations using (1) give

$$
\begin{aligned}
\left\|\pi_{1} v\right\|_{L_{2}\left(I_{j}\right)}^{2} & \leq\left|w_{j}\right|^{2}\left\|\psi_{j}\right\|_{L_{2}\left(I_{j}\right)}^{2}+\left|w_{j+1}\right|^{2}\left\|\psi_{j+1}\right\|_{L_{2}\left(I_{j}\right)}^{2}+2\left|w_{j}\right|\left|w_{j+1} \|\left(\psi_{j+1}, \psi_{j}\right)\right| \\
& \leq \frac{h_{j}}{r^{2}-1}\left(1+\frac{1}{r}\right)\left(\left|w_{j}\right|^{2}+\left|w_{j+1}\right|^{2}\right) \leq \frac{1}{r(r-1)}\left\|P_{j} W\right\|^{2} .
\end{aligned}
$$

To bound $\|F\|_{2}$, we note that

$$
\left|f_{i}\right|^{2} \leq\|v\|^{2} \frac{\left\|\psi_{i}\right\|_{L_{2}\left(I_{l}\right)}^{2}}{\left\|\psi_{i}\right\|^{4}}=\left(r^{2}-1\right)\|v\|^{2} \frac{h_{l}}{\left(h_{i-1}+h_{i}\right)^{2}}, \quad \text { for } i=l, l+1
$$

and hence

$$
\|F\|_{2}^{2}=\left(h_{l-1}+h_{l}\right)\left|f_{l}\right|^{2}+\left(h_{l}+h_{l+1}\right)\left|f_{l+1}\right|^{2} \leq\left(r^{2}-1\right)\|v\|^{2}\left(\frac{h_{l}}{h_{l-1}+h_{l}}+\frac{h_{l+1}}{h_{l}+h_{l_{1}}}\right) \leq 2\left(r^{2}-1\right)\|v\|^{2} .
$$

Altogether we obtain

$$
\begin{aligned}
\left\|\pi_{1} v\right\|_{L_{2}\left(I_{j}\right)} & \leq(r(r-1))^{-1 / 2}\left\|P_{j} W\right\| \leq r^{-|j-l|} r^{3 / 2}(r-1)^{-3 / 2}\|F\|_{2} \\
& \leq r^{-|j-l|} r^{3 / 2}(r+1)^{1 / 2}(r-1)^{-1} \sqrt{2}\|v\|=C_{r} r^{-|j-l|}\|v\|,
\end{aligned}
$$

which completes the proof.

A version of the following Lemma was shown in the quasiuniform case in [13]. 
Lemma 1.3. Under the assumption (1.6) we have

$$
\left\|\rho \delta_{h}^{x}\right\| \leq C h_{x}^{1 / 2}, \quad \text { for } x \in \Omega
$$

Proof. Let $x \in\left[x_{j}, x_{j+1}\right)$, recall that $h_{x}=h_{j}$. Then for any $\varphi \in \mathcal{C}_{0}^{\infty}\left(I_{l}\right)$ with $\|\varphi\|=1$ we have, using a local inverse estimate on $I_{j}$ and Lemma 1.2,

$$
\left(\delta_{h}^{x}, \varphi\right)=\left(\delta_{h}^{x}, P_{h} \varphi\right)=\left(P_{h} \varphi\right)(x) \leq C h_{j}^{-1 / 2}\left\|P_{h} \varphi\right\|_{L_{2}\left(I_{j}\right)} \leq C h_{j}^{-1 / 2} r^{-|l-j|} .
$$

Hence

$$
\left\|\delta_{h}^{x}\right\|_{L_{2}\left(I_{l}\right)}=\sup _{\substack{\varphi \in \mathcal{C}_{0}^{\infty}\left(I_{l}\right) \\\|\varphi\|=1}}\left(\delta_{h}^{x}, \varphi\right) \leq C h_{j}^{-1 / 2} r^{-|l-j|} .
$$

For $y \in I_{l}$ we also have, by (1.6),

$$
\rho(y)^{2}=|y-x|^{2}+h_{j}^{2} \leq\left(C \sum_{s=0}^{|l-j|} \alpha^{s} h_{j}\right)^{2}+h_{j}^{2} \leq C(|l-j|+1)^{2} \alpha^{2|l-j|} h_{j}^{2} .
$$

Hence, since $\alpha / r<1$,

$$
\left\|\rho \delta_{h}^{x}\right\|^{2} \leq \sum_{l=1}^{N} \sup _{I_{l}} \rho(y)^{2}\left\|\delta_{h}^{x}\right\|_{L_{2}\left(I_{j}\right)}^{2} \leq C \sum_{l=1}^{N}(|l-j|+1)^{2} \alpha^{2|l-j|} h_{j} r^{-2|l-j|} \leq C h_{j} \sum_{s \geq 0}(s+1)^{2}(\alpha / r)^{2 s}=C h_{j},
$$

which shows the Lemma.

Lemma 1.4. Under the assumptions from the beginning of this section we have

$$
|R(G, G)| \leq \frac{C}{(1+|\lambda|)^{3 / 2}}+\frac{C\left\|(\rho G)^{\prime}\right\|}{(1+|\lambda|)^{3 / 4}}, \quad \text { for } x \in \Omega, \quad \lambda \in \Sigma .
$$

Proof. We find at once

$$
R(G, G)=A(\rho G, \rho G)-A\left(\rho^{2} G, G\right)=\left(a \rho^{\prime} G, \rho^{\prime} G\right)-\left(b \rho^{\prime} G, \rho G\right)-2 \operatorname{Im}\left(a \rho^{\prime} G,(\rho G)^{\prime}\right),
$$

and hence, since $\left|\rho^{\prime}\right| \leq 1$,

$$
|R(G, G)| \leq C\|G\|^{2}+C\|G\|\left\|(\rho G)^{\prime}\right\| .
$$

The Lemma now follows by (1.10).

Lemma 1.5. Under the assumptions from the beginning of this section we have

$$
\left|A\left(\rho^{2} G-P_{h}\left(\rho^{2} G\right), G\right)\right| \leq \frac{C}{(1+|\lambda|)^{1 / 4}}\|\rho G\|, \quad \text { for } x \in \Omega, \quad \lambda \notin \Sigma_{\delta} .
$$

Proof. We set $\zeta=\rho^{2} G-R_{h}\left(\rho^{2} G\right)$ where $R_{h}$ is the $H_{0}^{1}$-projection onto $S_{h}$. Then $\rho^{2} G-P_{h}\left(\rho^{2} G\right)=\left(I-P_{h}\right) \zeta$. We have from Theorem 2 in $[5]$

$$
\left\|\left(\rho^{2} G-P_{h}\left(\rho^{2} G\right)\right)^{\prime}\right\|=\left\|\left(\left(I-P_{h}\right) \zeta\right)^{\prime}\right\| \leq C\left\|\zeta^{\prime}\right\| .
$$

Therefore

$$
\left|A\left(\rho^{2} G-P_{h}\left(\rho^{2} G\right), G\right)\right| \leq C\left\|\left(\rho^{2} G-P_{h}\left(\rho^{2} G\right)\right)^{\prime}\right\|\left\|G^{\prime}\right\| \leq \frac{C}{(1+|\lambda|)^{1 / 4}}\left\|\zeta^{\prime}\right\| .
$$


It is well known that, since we are in the one-dimensional case, $R_{h} u\left(x_{i}\right)=u\left(x_{i}\right)$ for all $i$. We consider now a subinterval $I_{j}$ and set $\rho_{j}=\rho\left(x_{j}\right)$. Noting that $\rho / \rho_{j}$ is bounded above and below on $I_{j}$ we have

$$
\left\|\zeta^{\prime}\right\|_{L_{2}\left(I_{j}\right)}=\left\|\left(\left(I-R_{h}\right)\left(\left(\rho^{2}-\rho_{j}^{2}\right) G\right)\right)^{\prime}\right\|_{L_{2}\left(I_{j}\right)} \leq C \rho_{j}\|G\|_{L_{2}\left(I_{j}\right)}+C \rho_{j} h_{j}\left\|G^{\prime}\right\|_{L_{2}\left(I_{j}\right)} \leq C\|\rho G\|_{L_{2}(I)} .
$$

Taking square and summing, this shows

In view of (1.17) this completes the proof.

$$
\left\|\zeta^{\prime}\right\| \leq C\|\rho G\|
$$

To continue the proof of Theorem 1 we set $\mu=(1+|\lambda|)^{-1 / 2}$ and obtain, using Lemmas $1.3,1.4$ and 1.5 , for $F$ defined in (1.12),

$$
|F| \leq\left|A\left(\rho^{2} G-P_{h}\left(\rho^{2} G\right), G\right)\right|+\|\rho G\|\|\rho \delta\|+|R(G, G)| \leq C\left(\mu^{1 / 2}+h_{x}^{1 / 2}\right)\|\rho G\|+C \mu^{3}+C \mu^{3 / 2}\left\|(\rho G)^{\prime}\right\| .
$$

Using (1.13) we deduce

$$
\begin{aligned}
\mu^{-2}\|\rho G\|^{2}+\left\|(\rho G)^{\prime}\right\|^{2} & \leq C\left(\mu^{1 / 2}+h_{x}^{1 / 2}\right)\|\rho G\|+C \mu^{3}+C \mu^{3 / 2}\left\|(\rho G)^{\prime}\right\| \\
& \leq \frac{1}{2} \mu^{-2}\|\rho G\|^{2}+C \mu^{2}\left(\mu+h_{x}\right)+C \mu^{3}+\left\|(\rho G)^{\prime}\right\|^{2} .
\end{aligned}
$$

Therefore

Using the estimate (1.11) we obtain

$$
\|\rho G\| \leq C \mu^{2}\left(h_{x}+\mu\right)^{1 / 2}
$$

$$
\|(\rho+\mu) G\| \leq C \mu^{2}\left(h_{x}+\mu\right)^{1 / 2} .
$$

Noting that

we finally have

$$
\left\|(\rho+\mu)^{-1}\right\|^{2} \leq 2 \int_{0}^{1} \frac{\mathrm{d} y}{y^{2}+h_{x}^{2}+\mu^{2}} \leq C\left(h_{x}+\mu\right)^{-1},
$$

which completes the proof.

$$
\|G\|_{L_{1}} \leq\left\|(\rho+\mu)^{-1}\right\|\|(\rho+\mu) G\| \leq C \mu^{2}=C(1+|\lambda|)^{-1},
$$

As a consequence of Theorem 1 we may conclude that $-A_{h}$ generates an analytic semigroup $E_{h}(t)=\mathrm{e}^{-A_{h} t}$, the solution operator of the semidiscrete problem

$$
\left(u_{h, t}, \chi\right)+A\left(u_{h}, \chi\right)=0 \quad \text { for } \chi \in S_{h}, t>0, \quad \text { with } u_{h}(\cdot, 0)=v_{h} \quad \text { in } \Omega,
$$

associated with the parabolic equation with elliptic operator $A$, and that stability and smoothing estimates as in (0.4) hold, this time without the logarithmic factor $\ell_{h}$, but with an exponentially growing factor $\mathrm{e}^{c_{1} t}$ if $c_{1}>0$ in $(1.2)$.

\section{THE TWO-DIMENSIONAL CASE}

In this section we consider the elliptic operator

$$
A u=-\operatorname{div}(a \nabla u)+\vec{b} \cdot \nabla u+c u, \quad \text { in } \Omega \subset \mathbb{R}^{2},
$$

with $a, \vec{b}, c$ bounded real-valued, and $a(x) \geq a_{0}>0$ in $\Omega$. This time we set

$$
A(u, w)=\int_{\Omega}(a \nabla u \cdot \nabla \bar{w}+\vec{b} \cdot \nabla u \bar{w}+c u \bar{w}) \mathrm{d} x,
$$


and note that there are $c_{0}>0, c_{1}, c_{2}, c_{3} \in \mathbb{R}$ such that

$$
c_{0}\|\nabla w\|^{2}-c_{1}\|w\|^{2} \leq \operatorname{Re} A(w, w) \leq c_{2}\|\nabla w\|^{2} \quad \text { and } \quad|\operatorname{Im} A(w, w)| \leq c_{3}\|\nabla w\|\|w\|, \quad \forall w \in H_{0}^{1} .
$$

The numerical range $W(A)$ is defined as in (1.3), and again (1.4) holds. As earlier we choose a closed subset $\Sigma \subset \mathbb{C}$ such that $\Sigma \cap \mathcal{P}=\emptyset$ and $d(\lambda, \mathcal{P}) \geq c(1+|\lambda|)$ for $\lambda \in \Sigma$.

We now consider triangulations $\mathcal{T}_{h}$ and the corresponding finite dimensional spaces $S_{h}$ consisting of piecewise polynomials of degree $r-1 \geq 1$, as defined in Introduction. We shall show the following resolvent estimate for the discrete version $A_{h}: S_{h} \rightarrow S_{h}$ of the operator $A$ in (2.1).

Theorem 2. Let the conditions on $\Omega$ and $\left\{\mathcal{T}_{h}\right\}$ from the introduction hold, in particular (0.6) and (0.7) with some $\alpha, \beta \geq 1$, and let

$$
\alpha^{2} \beta \gamma<1
$$

with $\gamma=\gamma_{r}$ as in Lemma 0.1. Then we have

$$
\left\|\left(\lambda I-A_{h}\right)^{-1} v_{h}\right\|_{\mathcal{C}} \leq \frac{C \ell_{h}^{1 / 2}}{1+|\lambda|}\left\|v_{h}\right\|_{\mathcal{C}}, \quad \forall v_{h} \in S_{h}, \quad \lambda \in \Sigma,
$$

where, as above, $\ell_{h}=\max \left(1, \log \left(1 / h_{\text {min }}\right)\right)$ with $h_{\min }=\min _{\tau \in \mathcal{T}_{h}} h_{\tau}$.

For $x \in \Omega$ fixed we will use the adjoint discrete Green's function

$$
G_{h}^{x}(y, \bar{\lambda})=\left(\left(\bar{\lambda} I-A_{h}^{*}\right)^{-1} \delta_{h}^{x}\right)(y) \quad \text { for } \lambda \in \Sigma,
$$

where $\delta_{h}^{x} \in S_{h}$ is the discrete delta-function defined by

$$
\left(\chi, \delta_{h}^{x}\right)=\chi(x), \quad \forall \chi \in S_{h} .
$$

As in Section 1 we have

$$
\left(\left(\lambda I-A_{h}\right)^{-1} \chi\right)(x)=\left(\chi, G_{h}^{x}(., \bar{\lambda})\right), \quad \forall \chi \in S_{h},
$$

and to prove the Theorem it suffices to show

$$
\left\|G_{h}^{x}(., \bar{\lambda})\right\|_{L_{1}} \leq \frac{C \ell_{h}^{1 / 2}}{1+|\lambda|}, \quad \text { for } \lambda \in \Sigma, x \in \Omega .
$$

We obtain in the same way as for Lemma 1.1.

Lemma 2.1. There is a constant $C=C_{\Sigma}$ such that, for $v \in H_{0}^{1}$ and $\lambda \in \Sigma$,

$$
\text { if } \quad \lambda\|v\|^{2}-A(v, v)=F, \quad \text { then } \quad(1+|\lambda|)\|v\|^{2}+\|\nabla v\|^{2} \leq C|F| .
$$

We note that, writing for brevity $G=G_{h}^{x}(\cdot, \bar{\lambda})$ for $x \in \Omega, \lambda \in \Sigma$,

$$
\lambda(\chi, G)-A(\chi, G)=\left(\chi, \delta_{h}^{x}\right)=\chi(x), \quad \forall \chi \in S_{h} .
$$

Choosing $\chi=G$ and using Lemma 2.1 we obtain

$$
(1+|\lambda|)\left|\|G\|^{2}+\|\nabla G\|^{2} \leq C\right| G(x) \mid \leq C\|G\|_{\mathcal{C}} \leq C \ell_{h}^{1 / 2}\|\nabla G\| .
$$

This yields, with $\mu:=(1+|\lambda|)^{-1 / 2}$,

$$
\|\nabla G\| \leq C \ell_{h}^{1 / 2} \quad \text { and } \quad\|G\| \leq C \mu \ell_{h}^{1 / 2} .
$$


Remark. It appears that the continuous Green's function $g=g^{x}(., \bar{\lambda})$ satisfies $\|\nabla g\|=\infty$ and $\|g\| \leq C \mu$. For $A=-\Delta$ this can be shown by an argument which starts with an explicit formula for the Green's function when $\Omega=\mathbb{R}^{2}$, and the conclusion should hold also for more general operators $A$. Thus the first estimate in (2.7) may be considered as satisfying, but an improvement of the second estimate to $\|G\| \leq C \mu$ might be possible and would show Theorem 2 without a logarithmic factor.

Now we will deduce the $L_{1}$ estimate (2.4) from the estimate of $\|G\|$. For $\lambda$ bounded this follows directly from the inequality $\|G\|_{L_{1}} \leq C\|G\|$. We now turn to larger values of $\lambda \in \Sigma$. With the given point $x \in \Omega_{h}$ we associate a triangle $\tau$ (arbitrarily if $x$ is on an edge) such that $x \in \tau$ and set $h_{x}=h_{\tau}$. We then use the weight function

$$
\rho(y)=\rho_{h}^{x}(y)=\left(|x-y|^{2}+h_{x}^{2}\right)^{1 / 2},
$$

and note that $\rho^{2}$ is a quadratic polynomial. We have

$$
\|G\|_{L_{1}} \leq\left\|\left(\rho^{2}+\mu^{2}\right)^{-1}\right\|\left\|\left(\rho^{2}+\mu^{2}\right) G\right\| \leq \frac{C}{h_{x}+\mu}\left\|\left(\rho^{2}+\mu^{2}\right) G\right\|,
$$

where the second inequality follows from

$$
\left\|\left(\rho^{2}+\mu^{2}\right)^{-1}\right\|^{2} \leq 2 \pi \int_{0}^{\infty} \frac{r \mathrm{~d} r}{\left(r^{2}+h_{x}^{2}+\mu^{2}\right)^{2}}=\frac{\pi}{h_{x}^{2}+\mu^{2}} .
$$

We shall show that

$$
\left\|\rho^{2} G\right\| \leq C \mu^{2}\left(h_{x}+\mu+\|G\|\right)
$$

and therefore

$$
\|G\|_{L_{1}} \leq C \mu(\mu+\|G\|)
$$

Using the second inequality in (2.7), this completes the proof of (2.4) and hence of the theorem.

For the proof of (2.9) we consider the expression

$$
\lambda\left\|\rho^{m} G\right\|^{2}-A\left(\rho^{m} G, \rho^{m} G\right)=\lambda\left(\rho^{2 m} G, G\right)-A\left(\rho^{2 m} G, G\right)-R_{m}(G, G),
$$

where $m=1$ or 2 , and

$$
R_{m}(G, G)=A\left(\rho^{m} G, \rho^{m} G\right)-A\left(\rho^{2 m} G, G\right) .
$$

After subtraction by $(2.5)$ with $\chi=P_{h}\left(\rho^{2 m} G\right)$, this yields

$$
\lambda\left\|\rho^{m} G\right\|^{2}-A\left(\rho^{m} G, \rho^{m} G\right)=F_{m},
$$

where

$$
F_{m}=-A\left(\rho^{2 m} G-P_{h}\left(\rho^{2 m} G\right), G\right)-\left(\rho^{2 m} G, \delta_{h}^{x}\right)-R_{m}(G, G) .
$$

By Lemma 2.1 it follows from (2.10) that

$$
\mu^{-2}\left\|\rho^{m} G\right\|^{2}+\left\|\nabla\left(\rho^{m} G\right)\right\|^{2} \leq C\left|F_{m}\right| .
$$

To show (2.9) we will use this first for $m=1$ and then for $m=2$, together with the appropriate bounds for $F_{1}$ and $F_{2}$. The bounds needed for these functions will require the following Lemmas, which are analogous to those used in the one-dimensional case.

Lemma 2.2. Under the assumption $\alpha^{2} \beta \gamma<1$ we have

$$
\left\|\rho \delta_{h}^{x}\right\| \leq C \quad \text { and } \quad\left\|\rho^{2} \delta_{h}^{x}\right\| \leq C h_{x}, \quad \text { for } x \in \Omega
$$


Lemma 2.3. Under the assumptions of Theorem 2 we have, for $x \in \Omega, \chi \in S_{h}$.

$$
\left|A\left(\rho^{2} \chi-P_{h}\left(\rho^{2} \chi\right), \chi\right)\right| \leq C\left(\|\nabla(\rho \chi)\|\|\chi\|+\|\chi\|^{2}\right)
$$

and

$$
\left|A\left(\rho^{4} \chi-P_{h}\left(\rho^{4} \chi\right), \chi\right)\right| \leq C(\|\nabla(\rho \chi)\|+\|\chi\|)\left\|\rho^{2} \chi\right\| .
$$

Assuming that these lemmas have been proved, we are now ready for the proof of our main result. We first remark that

$$
R_{m}(G, G)=m^{2}\left(a \rho^{m-1} G \nabla \rho, \rho^{m-1} G \nabla \rho\right)-m\left(\vec{b} \cdot \nabla \rho \rho^{m-1} G, \rho^{m} G\right)+2 m \operatorname{Im}\left(a \nabla\left(\rho^{m} G\right), \rho^{m-1} G \nabla \rho\right) .
$$

Using that $|\nabla \rho| \leq 1$ in $\Omega$, this implies

$$
\left|R_{m}(G, G)\right| \leq C\left(\left\|\nabla\left(\rho^{m} G\right)\right\|\left\|\rho^{m-1} G\right\|+\left\|\rho^{m-1} G\right\|^{2}\right) .
$$

We now take $m=1$ in (2.12) and use (2.11) to obtain

$$
\mu^{-2}\|\rho G\|^{2}+\|\nabla(\rho G)\|^{2} \leq C\left|F_{1}\right| \leq C\left(\left|A\left(\rho^{2} G-P_{h}\left(\rho^{2} G\right), G\right)\right|+\left|\left(\rho G, \rho \delta_{h}^{x}\right)\right|+\left|R_{1}(G, G)\right|\right) .
$$

Using Lemma 2.2, (2.13), and (2.15), we get

$$
\mu^{-2}\|\rho G\|^{2}+\|\nabla(\rho G)\|^{2} \leq C\left(\|\nabla(\rho G)\|\|G\|+\|G\|^{2}+\|\rho G\|\right) \leq \frac{1}{2}\left(\|\nabla(\rho G)\|^{2}+\mu^{-2}\|\rho G\|^{2}\right)+C\left(\mu^{2}+\|G\|^{2}\right),
$$

which shows

$$
\mu^{-2}\|\rho G\|^{2}+\|\nabla(\rho G)\|^{2} \leq C(\mu+\|G\|)^{2},
$$

and hence

$$
\|\rho G\| \leq C \mu(\mu+\|G\|) \quad \text { and } \quad\|\nabla(\rho G)\| \leq C(\mu+\|G\|) .
$$

We now take $m=2$ in (2.12) to find

$$
\mu^{-2}\left\|\rho^{2} G\right\|^{2}+\left\|\nabla\left(\rho^{2} G\right)\right\|^{2} \leq C\left|F_{2}\right| \leq C\left(\left|A\left(\rho^{4} G-P_{h}\left(\rho^{4} G\right), G\right)\right|+\left|\left(\rho^{2} G, \rho^{2} \delta_{h}^{x}\right)\right|+\left|R_{2}(G, G)\right|\right) .
$$

Hence, using Lemma 2.2, (2.14), and (2.15) with $m=2$,

$$
\begin{aligned}
\mu^{-2}\left\|\rho^{2} G\right\|^{2}+\left\|\nabla\left(\rho^{2} G\right)\right\|^{2} & \leq C\left(\left(\|\nabla(\rho G)\|+\|G\|+h_{x}\right)\left\|\rho^{2} G\right\|+\left\|\nabla\left(\rho^{2} G\right)\right\|\|\rho G\|+\|\rho G\|^{2}\right) \\
& \leq \frac{1}{2} \mu^{-2}\left\|\rho^{2} G\right\|^{2}+\left\|\nabla\left(\rho^{2} G\right)\right\|^{2}+C \mu^{2}\left(\|\nabla(\rho G)\|+\|G\|+h_{x}\right)^{2}+C\|\rho G\|^{2} .
\end{aligned}
$$

Using now (2.17) this yields

$$
\mu^{-2}\left\|\rho^{2} G\right\|^{2} \leq C \mu^{2}\left(h_{x}+\mu+\|G\|\right)^{2},
$$

and completes the proof of (2.9). It now only remains to prove Lemmas 2.2 and 2.3 .

Proof of Lemma 2.2. Let $\tau \in Q_{j}\left(\tau_{0}\right)$. Then for any $\varphi \in \mathcal{C}_{0}^{\infty}(\tau)$ with $\|\varphi\|=1$ we have, using Lemma 0.1,

$$
\left(\delta_{h}^{x}, \varphi\right)=\left(\delta_{h}^{x}, P_{h} \varphi\right)=\left(P_{h} \varphi\right)(x) \leq C h_{\tau_{0}}^{-1}\left\|P_{h} \varphi\right\|_{L_{2}\left(\tau_{0}\right)} \leq C h_{\tau_{0}}^{-1} \gamma^{j} .
$$

Hence

$$
\left\|\delta_{h}^{x}\right\|_{L_{2}(\tau)}=\sup _{\substack{\varphi \in \mathcal{C}_{0}^{\infty}(\tau) \\\|\varphi\|=1}}\left(\delta_{h}^{x}, \varphi\right) \leq C h_{\tau_{0}}^{-1} \gamma^{j}
$$


For $y \in \tau$ we also have

$$
\rho(y)^{2}=|y-x|^{2}+h_{\tau_{0}}^{2} \leq\left(C \sum_{l=0}^{j} \alpha^{l} h_{\tau_{0}}\right)^{2}+h_{\tau_{0}}^{2} \leq C(j+1)^{2} \alpha^{2 j} h_{\tau_{0}}^{2} .
$$

Hence, since $\alpha^{2} \beta \gamma^{2}<\alpha^{2} \beta \gamma<1$,

$$
\begin{aligned}
\left\|\rho \delta_{h}^{x}\right\|^{2} & \leq \sum_{j \geq 0} \sup _{Q_{j}\left(\tau_{0}\right)} \rho(y)^{2}\left\|\delta_{h}^{x}\right\|_{Q_{j}\left(\tau_{0}\right)}^{2} \leq \sum_{j \geq 0} C\left((j+1)^{2} \alpha^{2 j} h_{\tau_{0}}^{2}\right)\left(n_{j}(\tau) h_{\tau_{0}}^{-2} \gamma^{2 j}\right) \\
& \leq C \sum_{j \geq 0}(j+1)^{2}\left(\alpha^{2} \beta \gamma^{2}\right)^{j}=C<\infty .
\end{aligned}
$$

Using now that $\alpha^{4} \beta \gamma^{2} \leq\left(\alpha^{2} \beta \gamma\right)^{2}<1$,

$$
\begin{aligned}
\left\|\rho^{2} \delta_{h}^{x}\right\|^{2} & \leq \sum_{j \geq 0} \sup _{Q_{j}\left(\tau_{0}\right)} \rho(y)^{4}\left\|\delta_{h}^{x}\right\|_{Q_{j}\left(\tau_{0}\right)}^{2} \leq C \sum_{j \geq 0}\left((j+1)^{4} \alpha^{4 j} h_{\tau_{0}}^{4}\right)\left(n_{j}(\tau) h_{\tau_{0}}^{-2} \gamma^{2 j}\right) \\
& \leq C \sum_{j \geq 0}(j+1)^{4}\left(\alpha^{4} \beta \gamma^{2}\right)^{j} h_{\tau_{0}}^{2}=C h_{\tau_{0}}^{2}
\end{aligned}
$$

which completes the proof.

Proof of Lemma 2.3. We first remark that, for $\chi \in S_{h}$,

$$
\begin{aligned}
\left|A\left(\rho^{2} \chi-P_{h}\left(\rho^{2} \chi\right), \chi\right)\right| & \leq C\left(\left\|\rho^{-1} \nabla\left(\rho^{2} \chi-P_{h}\left(\rho^{2} \chi\right)\right)\right\|(\|\rho \nabla \chi\|+\|\chi\|)+\left\|\rho^{2} \chi-P_{h}\left(\rho^{2} \chi\right)\right\|\|\chi\|\right) \\
& \leq C\left(\left\|\rho^{-1} \nabla\left(\rho^{2} \chi-P_{h}\left(\rho^{2} \chi\right)\right)\right\|(\|\nabla(\rho \chi)\|+\|\chi\|)+\|\chi\|^{2}\right) .
\end{aligned}
$$

In order to get (2.13) it suffices to show that

$$
\left\|\rho^{-1} \nabla\left(\rho^{2} \chi-P_{h}\left(\rho^{2} \chi\right)\right)\right\| \leq C\|\chi\| .
$$

Let $I_{h}$ be the standard Lagrange interpolant from $\mathcal{C}\left(\Omega_{h}\right)$ onto the continuous, piecewise polynomials of degree $r-1$ on the triangulation $\mathcal{T}_{h}$ of $\Omega_{h}$. We shall apply $I_{h}$ only to functions which vanish on $\partial \Omega_{h}$ and then extend the definition of $I_{h} v$ to $\Omega$ by setting $I_{h} v=0$ in $\Omega \backslash \Omega_{h}$, so that $I_{h} v \in S_{h}$. Recall that there exists a constant $K$ such that, for any $\tau \in \mathcal{T}_{h}$ and any polynomial $q$ of degree $\leq r+3$, we have $\left\|I_{h} q\right\|_{L_{2}(\tau)} \leq K\|q\|_{L_{2}(\tau)}$. We set $\zeta_{1}=\rho^{2} \chi-I_{h}\left(\rho^{2} \chi\right)$, and we clearly have $\rho^{2} \chi-P_{h}\left(\rho^{2} \chi\right)=\left(I-P_{h}\right) \zeta_{1}$. It therefore now suffices to show that

$$
\left\|\rho^{-1} \nabla \zeta_{1}\right\| \leq C\|\chi\| \quad \text { and }\left\|\rho^{-1} \nabla\left(P_{h} \zeta_{1}\right)\right\| \leq C\|\chi\|
$$

For each $\tau \in \mathcal{T}_{h}$, let $x_{\tau} \in \tau$ and $\rho_{\tau}=\rho\left(x_{\tau}\right)$, and note that $\rho_{\tau} / \rho$ is bounded above and below on $\tau$. We then have $\zeta_{1}=\left(\rho^{2}-\rho_{\tau}^{2}\right) \chi-I_{h}\left(\left(\rho^{2}-\rho_{\tau}^{2}\right) \chi\right)$. Since $\left(\rho^{2}-\rho_{\tau}^{2}\right) \chi$ is a polynomial of degree at most $r+1$ we may use an inverse property and the boundedness of $I_{h}$ in $L_{2}(\tau)$ to obtain

$$
\left\|\nabla \zeta_{1}\right\|_{L_{2}(\tau)} \leq C h_{\tau}^{-1}\left\|\zeta_{1}\right\|_{L_{2}(\tau)} \leq C h_{\tau}^{-1}\left\|\left(\rho^{2}-\rho_{\tau}^{2}\right) \chi\right\|_{L_{2}(\tau)} \leq C\|\rho \chi\|_{L_{2}(\tau)},
$$

and thus

$$
\left\|\rho^{-1} \nabla \zeta_{1}\right\|_{L_{2}(\tau)} \leq C\|\chi\|_{L_{2}(\tau)}
$$

The first inequality in (2.19) now follows by squaring and summing over the triangles. 
In order to show the second inequality in (2.19), we start with

$$
\left\|\rho^{-1} \nabla\left(P_{h} \zeta_{1}\right)\right\|_{L_{2}(\tau)} \leq C \rho_{\tau}^{-1} h_{\tau}^{-1}\left\|P_{h} \zeta_{1}\right\|_{L_{2}(\tau)} .
$$

Using Lemma 0.1 we obtain

$$
\left\|P_{h} \zeta_{1}\right\|_{L_{2}(\tau)} \leq C \sum_{\tau^{\prime} \in \mathcal{T}_{h}} \gamma^{\ell\left(\tau, \tau^{\prime}\right)}\left\|\zeta_{1}\right\|_{L_{2}\left(\tau^{\prime}\right)}
$$

As in (2.20) we have

$$
\left\|\zeta_{1}\right\|_{L_{2}\left(\tau^{\prime}\right)} \leq C h_{\tau^{\prime}}\|\rho \chi\|_{L_{2}\left(\tau^{\prime}\right)}
$$

and hence

$$
\left\|\rho^{-1} \nabla\left(P_{h} \zeta_{1}\right)\right\|_{L_{2}(\tau)} \leq C \sum_{\tau^{\prime} \in \mathcal{T}_{h}} \gamma^{\ell\left(\tau, \tau^{\prime}\right)}\left(\rho_{\tau^{\prime}} / \rho_{\tau}\right)\left(h_{\tau^{\prime}} / h_{\tau}\right)\|\chi\|_{L_{2}\left(\tau^{\prime}\right)} .
$$

We now note that

$$
h_{\tau^{\prime}} / h_{\tau} \leq C \alpha^{\ell\left(\tau, \tau^{\prime}\right)} \quad \text { and } \quad \rho_{\tau^{\prime}} / \rho_{\tau} \leq C l\left(\tau, \tau^{\prime}\right) \alpha^{l\left(\tau, \tau^{\prime}\right)} .
$$

Indeed, the second inequality follows from

$$
\frac{\rho_{\tau^{\prime}}}{\rho_{\tau}}=\frac{\sqrt{\left|x_{\tau^{\prime}}-x\right|^{2}+h_{x}^{2}}}{\sqrt{\left|x_{\tau}-x\right|^{2}+h_{x}^{2}}} \leq 1+\frac{\left|x_{\tau^{\prime}}-x_{\tau}\right|}{\sqrt{\left|x_{\tau}-x\right|^{2}+h_{x}^{2}}} \leq 1+C \frac{\left|x_{\tau^{\prime}}-x_{\tau}\right|}{h_{\tau}} \leq C \sum_{j=1}^{l\left(\tau, \tau^{\prime}\right)} \alpha^{j} .
$$

Thus

$$
\left\|\rho^{-1} \nabla\left(P_{h} \zeta_{1}\right)\right\|_{L_{2}(\tau)} \leq C \sum_{\tau^{\prime} \in \mathcal{T}_{h}} l\left(\tau, \tau^{\prime}\right)\left(\alpha^{2} \gamma\right)^{l\left(\tau, \tau^{\prime}\right)}\|\chi\|_{L_{2}\left(\tau^{\prime}\right)} .
$$

We now use the fact that if $M=\left(m_{j k}\right)$ is a symmetric matrix, then its norm, subordinate to the Euclidean norm, is bounded by $\max _{j} \sum_{k}\left|m_{j k}\right|$, and deduce, since $\alpha^{2} \beta \gamma<1$,

$$
\left\|\rho^{-1} \nabla\left(P_{h} \zeta_{1}\right)\right\| \leq C\left(\max _{\tau} \sum_{\tau^{\prime} \in \mathcal{T}_{h}} l\left(\tau, \tau^{\prime}\right)\left(\alpha^{2} \gamma\right)^{l\left(\tau, \tau^{\prime}\right)}\right)\|\chi\| \leq C \sum_{j=1}^{\infty} j\left(\alpha^{2} \beta \gamma\right)^{j}\|\chi\| \leq C\|\chi\| .
$$

We now turn to the proof of (2.14). We have

$$
\begin{aligned}
\left|A\left(\rho^{4} \chi-P_{h}\left(\rho^{4} \chi\right), \chi\right)\right| & \leq C\left(\left\|\rho^{-1} \nabla\left(\rho^{4} \chi-P_{h}\left(\rho^{4} \chi\right)\right)\right\|(\|\rho \nabla \chi\|+\|\rho \chi\|)+\left\|\rho^{4} \chi-P_{h}\left(\rho^{4} \chi\right)\right\|\|\chi\|\right) \\
& \leq C\left(\left\|\rho^{-1} \nabla\left(\rho^{4} \chi-P_{h}\left(\rho^{4} \chi\right)\right)\right\|(\|\nabla(\rho \chi)\|+\|\chi\|)+\left\|\rho^{4} \chi\right\|\|\chi\|\right) .
\end{aligned}
$$

In order to get (2.14) it suffices to show that

$$
\left\|\rho^{-1} \nabla\left(\rho^{4} \chi-P_{h}\left(\rho^{4} \chi\right)\right)\right\| \leq C\left\|\rho^{2} \chi\right\| .
$$

For this we now set $\zeta_{2}=\rho^{4} \chi-I_{h}\left(\rho^{4} \chi\right)$. This time $\rho^{4} \chi-P_{h}\left(\rho^{4} \chi\right)=\left(I-P_{h}\right) \zeta_{2}$, and it thus now suffices to show that

$$
\left\|\rho^{-1} \nabla \zeta_{2}\right\| \leq C\left\|\rho^{2} \chi\right\| \quad \text { and }\left\|\rho^{-1} \nabla\left(P_{h} \zeta_{2}\right)\right\| \leq C\left\|\rho^{2} \chi\right\| .
$$

Similarly to the above we find

$$
\left\|\rho^{-1} \nabla \zeta_{2}\right\|_{L_{2}(\tau)} \leq C \rho_{\tau}^{-1} h_{\tau}^{-1}\left\|\zeta_{2}\right\|_{L_{2}(\tau)} \leq C \rho_{\tau}^{-1} h_{\tau}^{-1}\left\|\left(\rho^{4}-\rho_{\tau}^{4}\right) \chi\right\|_{L_{2}(\tau)} \leq C\left\|\rho^{2} \chi\right\|_{L_{2}(\tau)} .
$$

The first inequality in (2.23) now follows by squaring and summing over $\mathcal{T}_{h}$.

It remains to show the second inequality in (2.23). We start with

$$
\left\|\rho^{-1} \nabla\left(P_{h} \zeta_{2}\right)\right\|_{L_{2}(\tau)} \leq C \rho_{\tau}^{-1} h_{\tau}^{-1}\left\|P_{h} \zeta_{2}\right\|_{L_{2}(\tau)}
$$


Using Lemma 0.1 we obtain

As above we have

$$
\left\|P_{h} \zeta_{2}\right\|_{L_{2}(\tau)} \leq C \sum_{\tau^{\prime} \in \mathcal{T}_{h}} \gamma^{\ell\left(\tau, \tau^{\prime}\right)}\left\|\zeta_{2}\right\|_{L_{2}\left(\tau^{\prime}\right)} .
$$

and hence, using (2.21)

$$
\left\|\zeta_{2}\right\|_{L_{2}\left(\tau^{\prime}\right)} \leq C h_{\tau^{\prime}} \rho_{\tau^{\prime}}\left\|\rho^{2} \chi\right\|_{L_{2}\left(\tau^{\prime}\right)}
$$

$$
\left\|\rho^{-1} \nabla\left(P_{h} \zeta_{2}\right)\right\|_{L_{2}(\tau)} \leq C \sum_{\tau^{\prime} \in \mathcal{T}_{h}} \gamma^{\ell\left(\tau, \tau^{\prime}\right)}\left(\rho_{\tau^{\prime}} / \rho_{\tau}\right)\left(h_{\tau^{\prime}} / h_{\tau}\right)\left\|\rho^{2} \chi\right\|_{L_{2}\left(\tau^{\prime}\right)} \leq C \sum_{\tau^{\prime} \in \mathcal{T}_{h}} l\left(\tau, \tau^{\prime}\right)\left(\alpha^{2} \gamma\right)^{l\left(\tau, \tau^{\prime}\right)}\left\|\rho^{2} \chi\right\|_{L_{2}\left(\tau^{\prime}\right)}
$$

Arguing as for $\zeta_{1}$, we deduce

$$
\left\|\rho^{-1} \nabla\left(P_{h} \zeta_{2}\right)\right\| \leq C\left(\max _{\tau} \sum_{\tau^{\prime} \in \mathcal{T}_{h}} l\left(\tau, \tau^{\prime}\right)\left(\alpha^{2} \gamma\right)^{l\left(\tau, \tau^{\prime}\right)}\right)\left\|\rho^{2} \chi\right\| \leq C \sum_{j=1}^{\infty} j\left(\alpha^{2} \beta \gamma\right)^{j}\left\|\rho^{2} \chi\right\| \leq C\left\|\rho^{2} \chi\right\|,
$$

which completes the proof.

As in the one-dimensional case, Theorem 2 shows that $-A_{h}$ generates an analytic semigroup $E_{h}(t)=\mathrm{e}^{-A_{h} t}$, the solution operator of the semidiscrete analogue of the parabolic problem associated with the operator $A$, and that the corresponding stability and smoothing estimates hold, this time with a logarithmic factor $\ell_{h}^{1 / 2}$.

\section{REFERENCES}

[1] N.Yu. Bakaev, Maximum norm resolvent estimates for elliptic finite element operators. BIT 41 (2001) 215-239.

[2] N.Yu. Bakaev, S. Larsson and V. Thomée, Long-time behavior of backward difference type methods for parabolic equations with memory in Banach space. East-West J. Numer. Math. 6 (1998) 185-206.

[3] N.Yu. Bakaev, V. Thomée and L.B. Wahlbin, Maximum-norm estimates for resolvents of elliptic finite element operators. Math. Comp. 72 (2002) 1597-1610.

[4] P. Chatzipantelidis, R.D. Lazarov, V. Thomée and L.B. Wahlbin, Parabolic finite element equations in nonconvex polygonal domains. BIT (to appear).

[5] M. Crouzeix and V. Thomée, The stability in $L_{p}$ and $W_{p}^{1}$ of the $L_{2}$-projection onto finite element function spaces. Math. Comp. 48 (1987) 521-532.

[6] M. Crouzeix and V. Thomée, Resolvent estimates in $l_{p}$ for discrete Laplacians on irregular meshes and maximum-norm stability of parabolic finite difference schemes. Comput. Meth. Appl. Math. 1 (2001) 3-17.

[7] M. Crouzeix, S. Larsson and V. Thomée, Resolvent estimates for elliptic finite element operators in one dimension. Math. Comp. 63 (1994) 121-140.

[8] E.L. Ouhabaz, Gaussian estimates and holomorphy of semigroups. Proc. Amer. Math. Soc. 123 (1995) 1465-1474.

[9] A.H. Schatz, V. Thomée and L.B. Wahlbin, Maximum norm stability and error estimates in parabolic finite element equations. Comm. Pure Appl. Math. 33 (1980) 265-304.

[10] A.H. Schatz, V. Thomée and L.B. Wahlbin, Stability, analyticity, and almost best approximation in maximum-norm for parabolic finite element equations. Comm. Pure Appl. Math. 51 (1998) 1349-1385.

[11] H.B. Stewart, Generation of analytic semigroups by strongly elliptic operators. Trans. Amer. Math. Soc. 199 (1974) $141-161$.

[12] V. Thomée, Galerkin Finite Element Methods for Parabolic Problems. Springer-Verlag, New York (1997).

[13] V. Thomée and L.B. Wahlbin, Maximum-norm stability and error estimates in Galerkin methods for parabolic equations in one space variable. Numer. Math. 41 (1983) 345-371. 